\title{
Examination of Three Pyrolytic Reaction Schemes for Cellulosic Materials
}

\author{
INDREK S. WICHMAN and ADEDEJO B. OLADIPO \\ Department of Mechanical Engineering \\ Michigan State University \\ East Lansing, MI 48824-1226 \\ USA
}

\begin{abstract}
Three decomposition schemes for cellulose and wood are examined, the one-step engineering scheme, the three-step delinked scheme proposed by Shafizadeh and Chin and the three-step coupled scheme of Broido. The one-step scheme, though simple, can describe many behaviors observed in real problems. These include pyrolysis temperature variation with heating rate, fixed change of pyrolysis temperature with successive doubling of heating rate, and the influences of variable char yield. In addition, kinetic constants may be chosen that fit the one-step model predictions of the three-step schemes. Among the three-step schemes a strong case can be made for the superiority of the Broido scheme because it has coupled competition between the two main product pathways.
\end{abstract}

KEYWORDS: Asymptotic Analysis, Cellulose, Decomposition, Reaction Mechanism

\section{INTRODUCTION}

Wood is an extremely difficult material to characterize, hence much previous research has focused instead on cellulose. The rationale is that pure cellulose is a good model for wood, which itself is approximately $75 \%$ cellulosic, the remainder being primarily lignin. Intuitively, we expect that if an accurate and useful description of a relatively simple substance like cellulose cannot be found, our prospects for describing complex materials like wood are slim indeed. In reality, the importance of studying cellulose extends far beyond its use as a simplified substitute wood. Cellulose is in fact a major constituent material of most households and therefore represents an important fraction of the domestic fuel load threatened by fire.

In spite of the relative simplicity of cellulose, however, the models of its decomposition, or pyrolysis, have many forms, see Table 1. The simplest model is the one-step global or engineering model, see refs. 1-3. We note that many such one-step models [1-3] are actually used to calculate wood pyrolysis rates. Extensions of the simple thermal degradation model have recently been made [4] to include global oxidation of the cellulose plus global oxidation of the final char. Nevertheless, the fundamental form of the resultant model is qualitatively the same. Each reaction makes products in fixed ratios which must be determined experimentally. Clearly 
A more complex family of decomposition models than the one-step model has been developed by chemists. Their interest is primarily in describing the separate steps of the process [5-7], much as in gas-phase chemical kinetics. The simplest model is the three-step delinked model proposed by Shafizadeh and Chin [5] and used thereafter by numerous authors, some extending it to describe wood [8]. In this model the virgin cellulose decomposes directly into three different compounds, char, tar, and inert gases (see Table 1). Competition occurs between the separate steps, which are delinked. The only linking between char, tar, gases, and cellulose occurs through the mass conservation constraint, $\sum_{i=1}^{N} y_{i}=1$. A similar model was proposed by Lipska and Parker [9], although theirs, for which they never wrote model equations, is zeroth order in the initial cellulose unzipping step. However, a zeroth order decomposition step poses severe modeling difficulties, especially if the remaining steps are not zeroth order. Nevertheless, there is much valuable insight in Ref. 9 concerning the separate rates for the crystalline and amorphous regions, and the hypothesis that char and tar are formed after the cellulose has unzipped essentially to levoglucosan. However, without any model equations or kinetic constants it is difficult to proceed with an analysis based on their observations.

A three-step model that is at least qualitatively similar to the Lipska-Parker model is the mechanism of Broido [7,10]. This model, developed after many years of experimental research, is different from the delinked scheme in that the decomposition of cellulose into products occurs through processes which are coupled. Hence, a change in the rate of production of one component, say char, through increased $k_{c}$, is accompanied by a change in the rate of production of $y_{A}$, the active cellulose, which in turn changes the rate of production of tar and gases. It is this powerful statement of mass or atom conservation at the molecular level and its simplicity of expression, generality and capability for refinement that make the Broido scheme an attractive model. Interestingly, Shafizadeh, who originally postulated the delinked three-step scheme [5], later abandoned it (Ref. 6) in favor of one resembling Broido's even to the point of measuring the kinetic constants that we shall use in this article.

There are some drawbacks of the Broido scheme. For instance, there has apparently been no direct proof of the existence of active cellulose [10]. Hence there is room here for refinement. We note, however, that from the viewpoint of modeling this drawback is not serious. We know that decomposition does not occur through a single step like cellulose $\rightarrow$ active cellulose. In other words, the cellulose breaks down through a complex sequence of individual steps involving depolymerization, abstraction of various molecules and compounds, etc. But even if we did know the individual decomposition pathways, we should still like to represent all of them with a single step. Hence, the use of an active intermediate completely fulfills our modeling requirements.

The most complex family of cellulose degradation models are the various fundamental chemical models that attempt to isolate actual single steps. Although these basic studies may eventually provide insight into detailed cellulose oxidation mechanisms or the influences of various catalysts and impurities, they have not yet made a significant impact on the modeling problem. They shall not be further discussed in this work.

In what follows we shall focus only on the decomposition mechanisms. Hence, the cellulose 
sample is assumed infinitesimally thin thereby eliminating heat transfer and species transport effects [11]. The complicating influences of moisture are also not considered.

\section{DECOMPOSITION MODELS}

In this section, we examine the solutions of the three models shown in Table 1 for constanttemperature heating. We note that because a sample that is heated to a certain test temperature must pass through all intermediate temperatures, constant-temperature heating is, at best, a limiting case of variable-temperature heating.

TABLE 1. The three reaction schemes examined herein and the corresponding parameters

\begin{tabular}{|c|c|c|c|}
\hline Model & Kinetic Mechanism & Parameters & $\begin{array}{c}\text { Variable-Rate } \\
\text { Heating }\end{array}$ \\
\hline One-step & $\dot{y}=-k(y-\delta)$ & $\begin{array}{l}\delta=y_{c}(\infty) \\
k=A \exp (-E / R T)\end{array}$ & $i=-z f(\theta)$ \\
\hline $\begin{array}{l}\text { Shafizadeh } \\
\text {-Chin }\end{array}$ & $\begin{array}{l}\dot{y}_{C E L L}=-k_{C E L L} y_{C E L L} \\
\dot{y}_{c}=k_{g} y_{C E L L} \\
\dot{y}_{T}=k_{T} y_{C E L L} \\
\dot{y}_{g}=k_{g} y_{C E L L}\end{array}$ & $\begin{array}{l}k_{i}=A_{i} \exp \left(-E_{i} / R T\right), \\
\hat{i}=C E L L, c, T, g \\
k_{C E L L}=k_{c}+k_{T}+k_{g}\end{array}$ & $\begin{array}{l}\dot{y}_{C E L L}=-y_{C E L L} f_{C E L L}(\theta) \\
\dot{y}_{i}=\alpha_{i} y_{C E L L} f_{i}(\theta), \quad i=c, T \\
y_{g}=1-\left(y_{C E L L}+y_{c}+y_{T}\right)\end{array}$ \\
\hline Broido & $\begin{array}{l}\dot{y}_{C E L L}=-k_{C E L L} y_{C E L L} \\
\dot{y}_{A}=k_{C E L L} y_{C E L L}-\left(k_{c}+k_{T}\right) y_{A} \\
\dot{y}_{c}=\mu k_{c} y_{A} \\
\dot{y}_{T}=k_{T} y_{A} \\
\dot{y}_{g}=(1-\mu) / \mu k_{c} y_{A}\end{array}$ & $\begin{array}{l}k_{i}=A_{i} \exp \left(-E_{i} / R T\right), \\
i=C E L L, C, T \\
\mu=\text { max. char yield }\end{array}$ & $\begin{aligned} \dot{y}_{C E L L}=-y_{C E L L} f_{C E L L}(\theta) & (\theta) \\
\dot{y}_{A} & y_{C E L L} f_{C E L L}(\theta) \\
& \quad\left(\alpha_{f} f_{T}(\theta)+\alpha_{f} f_{c}(\theta)\right) y_{A} \\
\dot{y}_{c} & =\mu \alpha_{c} y_{A} f_{c}(\theta) \\
y_{T} & =\left(\alpha_{v} / \mu \alpha_{c}\right) y_{c}\end{aligned}$ \\
\hline
\end{tabular}

For the one-step model, we find that the normalized residual mass, $z=\left(y-y_{c}(\infty)\right) /\left(1-y_{c}(\infty)\right)$, is given by $z=e^{-\tau}$, where $\tau$ is the normalized time. We note that it is impossible to discriminate between the remaining separate components of the residual mass during decomposition unless additional formulae describing their relative proportions are given. Thus, as far as the solid residue problem is concerned, our one-step model is incomplete.

In order to solve the decomposition equations of the three-step delinked model efficiently, we 
define $\alpha_{i}=k_{i} / k_{C E L L}$ as nondimensional ratios of the kinetic rate constants. Then $y_{C E L L}=\exp \left(-k_{C E L L} t\right), y_{i}=\alpha_{i}\left(1-y_{C E L L}\right), \quad i=c, T, g$. If at time $t_{*}$ we should change the heating temperature stepwise from $T_{\text {test } 1}$ to $T_{\text {test } 2}$ we would find that $y_{C E L L}=y_{C E L L}\left(t_{*}\right) \exp \left[-k_{C E L L 2}\left(t-t_{*}\right)\right]$, $y_{c}=y_{c}\left(t_{*}\right)+\alpha_{c 2} y_{C E L L}\left(t_{*}\right)\left[1-y_{C E L L} / y_{C E L L}\left(t_{*}\right)\right]$, etc. Here $k_{C E L L ~ 2}, \alpha_{c 2}, \ldots$ are evaluated at $T_{\text {test } 2}$.

The ultimate char yield for this two-stage heating is $y_{c}(\infty)=y_{c}\left(t_{*}\right)+\alpha_{c 2} y_{C E L L}\left(t_{*}\right)=\alpha_{c 1}+\left(\alpha_{c 2}-\alpha_{c 1}\right) y_{C E L L}\left(t_{*}\right)$. The first term is the value if heating continued indefinitely at $T_{\text {test } 1}$. The second term represents the increment produced by changing $T_{\text {test }}$. It is negative when $T_{\text {test } 2}<T_{\text {test 1 }}$, positive when $T_{\text {test } 2}>T_{\text {test 1 }}$.

If the char yield varies, the tar and gas yields must also be modified. The yields of these products of decomposition depend strongly on the temperature. A useful measure is the crossover temperature, $T_{c r}$, for the char and tar reaction rates, given as the value of $T$ at which $k_{T}=k_{c}$, viz.,

$T_{c r}=\frac{\left(E_{T}-E_{c}\right) / R}{\ln \left(A_{T} / A_{c}\right)}$

When $T>T_{c r}$ we have $k_{T}>k_{c}$. Tar production dominates charring. When $T<T_{c r}$ the reverse is true. Normally, $T_{c r} \sim 300^{\circ} \mathrm{C}[10]$.

For the three-step Broido model, the equations of Table 1 are easily solved for constant test temperature. We obtain

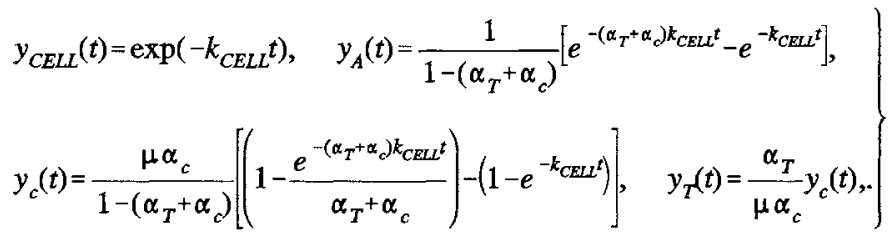

Note that $y_{C E L L}+y_{A}+y_{c}+y_{T}+y_{g}=1$ by mass conservation. Also, $y_{c}(\infty)=\mu /\left(1+\alpha_{l} / \alpha_{c}\right)$, where $\mu$ is the maximum char yield, which is attained in the limit $\alpha_{T} / \alpha_{c} \rightarrow 0$. This occurs when $T_{\text {test }}$ is significantly lower than $T_{c r}$, where $T_{c r}$ is given by Eq. (1). When the test temperature is changed stepwise the deductions are the same as with the three-step delinked model.

Now we shall demonstrate that it is possible to derive reasonable kinetic constants for the onestep scheme from the three-step schemes. Our attention is focused on the Broido scheme. As our principal measure we employ the normalized residual mass. For the one-step scheme this is simply $z$., as defined in the paragraph on the one-step model. For the Broido scheme the remaining mass is $y_{C E L L}+y_{A}+y_{c}$ if we count tars as volatiles that no longer belong to the solid. The normalized residual mass is $z=\left[\left(y_{C E L L}+y_{A}+y_{c}\right)-y_{c}(\infty)\right] /\left[1-y_{c}(\infty)\right]$. Upon substitution of Eqs. (2) into this formula we obtain $z=\left[e^{-\alpha, s}-\alpha e^{-s}\right] /(1-\alpha)$, where $\alpha=\alpha_{T}+\alpha_{c}$ and $s=k_{C E L L} t$.

In order to derive rate constants for the one-step scheme we fix two test temperatures, $T_{\text {test } 1}$ and $T_{\text {eest } 2}$ 
and calculate from the Broido $z$ - equation the values of $t_{\text {test } 1}$ and $t_{\text {test } 2}$ needed to reduce $z$ to $e^{-1}$. For the one-step scheme, $z=e^{-1}$ when $\tau=1$. Hence, $t_{\text {test }}=\left[A \exp \left(-E / R T_{\text {test }}\right)\right]^{-1}$. With the two sets of $T_{\text {test }}, t_{\text {test }}$ from the Broido scheme we calculate $A$ and $E$ values from

$E=R T_{\text {test } 1} \ln \mathrm{X} /(1-\Upsilon) ; \quad A=t_{\text {test } 1}^{-1} \mathrm{X}^{1 /(1-\Upsilon)}$.

where $\mathrm{X}=t_{\text {test } 1} / t_{\text {test } 2}$ and $\Upsilon=T_{\text {test } 1} / T_{\text {test } 2}$. Using the values for $A_{C E L L}, A_{c}, A_{T}, E_{C E L L}, E_{c}, E_{T}$, given in Ref. 10 and utilizing two test temperatures $T_{\text {test } 1}=550 \mathrm{~K}, T_{\text {test } 2}=850 \mathrm{~K}$ gives $t_{\text {test } 1}=15064 \mathrm{sec}$. and $t_{\text {test } 2}=2.72 \times 10^{-2} \mathrm{sec}$., yielding $E=40.9 \mathrm{kcal} / \mathrm{mole}$ and $A=1.24 \times 10^{12} \mathrm{sec}^{-1}$ as reasonable one-step parameters derived from the three step Broido scheme parameters. A test time comparison shows that near $500 \mathrm{~K}$ and $850 \mathrm{~K}$ the one-step formula is accurate. In between it may differ by up to $30 \%$.

Ideally, we should like to minimize the residual mass difference $z_{\text {BROIDo }}-z_{1-S T E P}=e^{-\alpha k_{C E L L} t}-\alpha e^{-k_{C E L} t} /(1-\alpha)-e^{-\tau}$ for each $T_{\text {test }}$ For a given $T_{\text {rest }}$ we fix $\alpha$ and $k_{C E L L}$, requiring a choice of $k$ for the one-step expression that minimizes the difference for all $t$. Note that for small $\alpha$, which occurs when $T_{\text {test }} \geq 600 K \doteq 300^{\circ} \mathrm{C}$, we have $z_{B R O I D O}-z_{1-S T E P} \approx \exp \left[-\left(k_{c}+k_{T}\right) t\right]-\exp [-k t]$ when $t \gg k_{C E L L}^{-1}$. The latter condition is easily satisfied in practice because $k_{C E L L}$ becomes very large as $T_{\text {test }}$ increases. The difference is zero when $k=k_{c}+k_{T}$. This reduces to $k \approx k_{T}$, when $T_{\text {test }}>T_{c r}$. Hence, for large $T_{\text {test }}$, the choice of parameters for the one-step model is largely determined by the volatilization step. We may then write $A \approx A_{T}, E \approx E_{T}$ in the one-step expression.

\section{VARIABLE-RATE HEATING}

Here we must augment the decomposition equations with an energy equation like $d T / d t=g(t)$, $T(0)=T_{o}$. This equation simply dictates the heating rate imposed on the sample. We define a characteristic heat-up time as $t_{H E A T}=\left(T_{M A X}-T_{0}\right) / g(0)$, where $T_{M A X}$ is the maximum sample temperature. Then we define the reduced temperature $\theta$, which varies between zero and one. With $\tau=t / t_{\boldsymbol{K} I N}$ we obtain the energy equation,

$\frac{d \theta}{d \tau}=D^{-1} G(\tau) ; \quad \theta(0)=0$

and the species equations shown in the rightmost column of Table 1 . The initial conditions are $z(0)=1, y_{C E L L}(0)=1$, and $y_{i}(0)=0$ for all the other species.

These equations are in standard form for a high-activation-energy-asymptotic (AEA) calculation. Because of the relative largeness of $E$ and the relative smallness of $T_{M A X}$, the $\beta_{i}$ will be at least twice as large as for usual gas-phase AEA calculations. Hence, AEA methods should be quite accurate. The $\beta_{i}$ are fairly insensitive to $T_{M A X}$ in the approximate range $300^{\circ} \mathrm{C} \leq T_{M A X} \leq 600^{\circ} \mathrm{C}$.

For large $\beta_{i}$ the chemical reaction is frozen until $\theta$ is close to unity. Here $T$ is near $T_{M A X}$. Hence, we define the rescaled nondimensional temperature $\theta=1-\theta / \beta$. For the one-step scheme the choice of $\beta$ is obvious, but for the three-step schemes we use $\beta=\beta_{C E L L}$, from the initiation step. We now postulate a one-to-one relationship between temperature and time, so Eq. (4) can be written as $d \theta / d \tau=D^{-1} G(\tau)=D^{-1} H(\theta), \theta(0)=0$. Note that $\theta=1$ at $\tau=\infty$ and $\theta=0$ at $\tau=0$. Hence, $H(0)=1$ and $H(1)=0$. The lowest-order equation for $\Theta$ is $d \Theta / d \tau=-\Omega \Theta+\ldots$, where 
$\Omega=|-d H(1) / d \theta| D^{-1}$. When $\Omega \gg 1$ the heat-up time is so short that the temperature rise is nearly instantaneous, i.e., $H(\theta) \rightarrow$ step function. One then essentially ignores the energy equation and puts $\theta=1$ in the species equations to recover the constant $-T$ solutions. When $\Omega<1$ the heat-up time is much larger than the chemical time; not much cellulose is left once the test condition $T=T_{M A X}$ is attained. This limit is experimentally undesirable. The third limit, $\Omega \sim O(1)$, contains features of the other two and therefore is the most interesting. To examine it we substitute $\theta=1-\theta / \beta$ into the species equations and let $\beta \rightarrow \infty$ to obtain $f_{i}(\theta) \doteq \exp \left(-\epsilon_{i} \Theta\right)$. We also transform the left-hand sides of the equations as $d y_{i} / d \tau=-\Omega \Theta d y / d \Theta$. The resulting systems of equations can then be solved, although the solution for the three-step models is quite complicated. A considerable mathematical simplification is obtained by putting $\epsilon_{c}=\epsilon_{T}=1$. This, in essence, reduces our analysis to one of the mathematical character of the solution. The results are:

One-step:

$$
y=y_{c}(\infty)+\left(1-y_{c}(\infty)\right) e^{-v} ; \quad z_{1-S T E P}=e^{-v}
$$

3-step delinked: $\quad y_{C E L L}=e^{-v} ; y_{i}=\alpha_{i}\left(1-y_{C E L L}\right), i=c, T, g$;

$$
z_{3 D L} \equiv\left[\left(y_{C E L L}+y_{c}\right)-y_{c}(\infty)\right] /\left[1-y_{c}(\infty)\right]=e^{-v}
$$

3-step Broido:

$$
\begin{aligned}
& y_{C E L L}=e^{-v} ; y_{A}=\frac{1}{\alpha-1}\left[e^{-v}-e^{-\alpha v}\right] ; \quad y_{c}=\frac{\mu \alpha_{c}}{\alpha-1}\left[1-e^{-v}-\left(\frac{1-e^{-\alpha v}}{\alpha}\right) ; \quad y_{T}=\frac{\alpha_{v}}{\mu \alpha_{c}} y_{c}\right. \\
& \left.z_{B R O I D O}=\left[\left(y_{C E L L}+y_{A}+y_{c}\right)-y_{c}(\infty)\right] / 1-y_{c}(\infty)\right]=e^{-v}+\left(e^{-\alpha v}-e^{-v}\right) /(1-\alpha)
\end{aligned}
$$

where $v=\Omega^{-1} \int_{\Theta}^{\infty} x^{-1} \exp (-x) d x=\Omega^{-1} E_{1}(\Theta)$.

Some observations can be made from Eqs. (5). First, the residual mass decays at the same rate for the one-step and three-step delinked schemes, but for the Broido scheme decays more slowly, by the amount $\left(e^{-\alpha v}-e^{-v}\right) /(1-\alpha)$. Hence, the ratios $z_{1-S T E P} / z_{B R O I D O}-z_{3 D I} / z_{B R O I D O}$ are always $\leq 1$. At $T=T_{M A X}$ we have $v \rightarrow \infty$ so that $z_{1-\text { step }} / z_{B R O I D O} \rightarrow 0$ when $0<\alpha<1$. In other words, more residual mass remains to be pyrolyzed in the Broido mechanism. Second, we observe that for $z$, the only scheme that shows sensitivity to the various decomposition steps is the Broido scheme. This occurs through the parameter $\alpha$. The qualitative nature of the dependence does not change when $\alpha$ varies between zero and one. Finally, in the three-step delinked model we have two independent parameters, since $\alpha_{c}+\alpha_{T}+\alpha_{g}=1$ whereas in the Broido scheme we have three independent parameters. This implies a greater variety of behaviors for the Broido scheme.

\section{CONSTANT HEATING RATE, $T=T_{o}+g_{o} t$}

Many experiments are conducted at constant heating rate. Although the upper limit temperature is unbounded, in practice it suffices to discontinue heating once there is no further weight loss. Although the meaning of $T_{M A X}$ is slightly obscured, the notion of a $T_{M A X}$ remains relevant. Hence, our nondimensionalization of the previous section is still employed here.

Let us consider first the one-step reaction. Into Eq. (4) we put $G(\tau)=1$, giving $\theta=D^{-1} \tau$ for the temperature distribution. Substitution into the one-step species equation gives $d z / z=-D f(\theta) d \theta$ subject to $z=1$ at $\theta=0$. Integration gives

$z=\exp [-(D / \beta) I(\Theta ; \beta, \sigma)] ; \quad$ where $I(\Theta ; \beta, \sigma)=\int_{\Theta}^{\beta} e^{\frac{-u}{1-\sigma u / \beta}} d u ; \quad \Theta=\beta(1-\theta)$. 
When $\beta$ is large we have $I(\Theta ; \beta, \sigma) \cong I(\Theta ; \infty, \sigma)=e^{-\Theta}$. Consequently we obtain the approximate result,

$z=\exp \left[-(D / \beta) e^{-\theta}\right]$.

It can be shown, after a rather lengthy analysis, that for successive values of the heating rate, $g_{o_{i+1}} / g_{o_{i}}=2$, the change in temperature, $\Delta T_{i}=T_{i+1}-T_{i}$, at an identical stage of decomposition is given by

$\Delta T_{i} \approx \frac{T_{\text {MAX }_{i}}}{E / R T_{M A X}} \frac{\ln 2}{\left[1+2\left(1-\gamma_{i}\right)\right]}$,

where $\gamma_{i} \equiv T / T_{M A X}$ is a number smaller than 1. If $\gamma_{i} \approx$ constant we see immediately that $\partial\left(\Delta T_{i}\right) / \partial T_{M A X_{i}}>0$, indicating that the temperature difference increases with the heating rate. Note that $T_{M A X_{i}}$ is the value of $T$ at which no more mass loss occurs. It increases monotonically with $g_{0}$. Eq. (7) shows that as $E / R T_{M A X_{i}} \rightarrow \infty, \Delta T_{i} \rightarrow 0$, and the ignition-temperature concept is recovered. An estimate for $\Delta T_{i}$ can be made from Fig. 1. We use the results for the normalized residual solid mass, $z$, versus $T$ for various heating rates for the Broido mechanism. The curves are very similar to those obtained from the one-step scheme, in that the curves appear to be displaced by nearly fixed amounts and that at the higher heating rates the gaps seem to widen slightly. For our comparison we employ the last two curves in Fig. 1 with $g_{o}=80^{\circ} \mathrm{C} / \mathrm{min}$ and $g_{o}=160^{\circ} \mathrm{C} / \mathrm{min}$. We use $E \sim 40 \mathrm{kcal} / \mathrm{mole}$ and choose the decomposition level as $z=0.5$. We see that $T_{i}$, the value of $T$ when $g_{o}=80^{\circ} \mathrm{C} / \mathrm{min}$ and $z=0.5$, is approximately $465^{\circ} \mathrm{C}$. we estimate that $T_{M A X} \approx 525^{\circ} \mathrm{C}$. Hence $\gamma_{i}=0.8, E / R T_{M A X} \propto 25$ and $\Delta T \approx 15^{\circ} \mathrm{C}$, which is very close to the $\Delta T$ shown in Fig. 1.

Consequently, we have demonstrated the general absence of a characteristic pyrolysis temperature $T_{p}$. Assumption of a pyrolysis temperature is common in fire propagation models. The $T_{n}$ - concept appears to be accurate when $E / R T_{M A X} \curvearrowright(1)$.

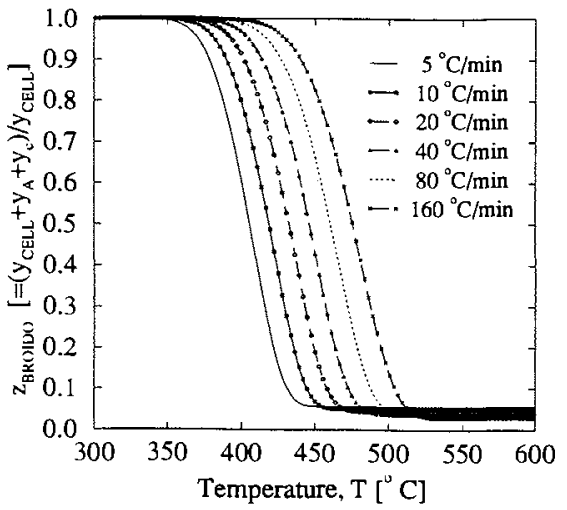

Figure 1. Numerical solution of the Broido model for various fixed heating rates $g_{o}$

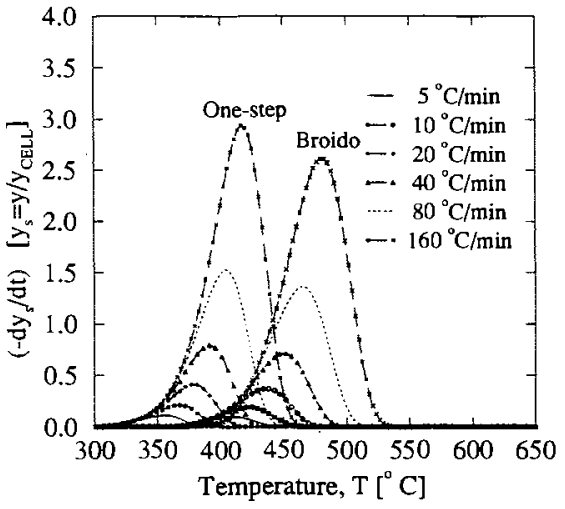

Figure 2. Plots of $d y / d \tau$ versus $T$ for the one-step and Broido models for various fixed heating rate $g_{0}$ 
As already mentioned, the fixed heating rate curves for the Broido and one-step mechanisms are qualitatively similar. In fact, plots of $d y / d \tau$ versus $T$, where $y$ is the residual mass, $y_{C E L L}+y_{C}+y_{A}$, produce only a single peak (see Fig. 2) thereby indicating --according to Ref. 4-a global one-step mechanism. But the Broido mechanism is not a one-step process, it is a threestep process. Hence, we are led to question the generality of deductions made from weight loss rate measurements.

\section{DISCUSSION}

There has been, and continues to be, much interest in the pyrolysis and combustion of wood and other cellulosic materials. Typical technological areas of interest are wood gasifiers, waste incinerators, and fire safety of wood and wood products. A recent issue of importance is the fire safety of organic composites consisting of wood fiber and a polymeric filler such as high-density polyethylene (HDPE). In the realm of modeling, there are few useful and reliable models for standard problems like flame spread over cellulosics, or the flame/smolder transition. The crucial, indeed central, missing link in these models is a robust, accurate description of the solid phase decomposition and pyrolysis.

Engineers have for many decades employed a relatively simple global one-step decomposition reaction, see the first row of Table 1 . We have demonstrated herein that this model has positive features, among them the capability of reproducing several global features of more complicated schemes, as shown in Figs. 1 and 2. Hence, processes like weight-loss rate can be reasonably effectively modeled, and the basic physics of the constant heating rate can be explained. Nevertheless the global scheme suffers from a serious built-in flaw. The decomposition products are formed in fixed ratios. That is, if we consider char and volatiles as our products, for example, we can generally say only that their ratios are fixed. The only remedy is to painstakingly develop experimentally measured correlations that produce product ratios which vary with temperature and the gas-phase environment. But in this case, it would seem to be more advantageous to simply develop a more realistic model.

The three-step models present a viable solution to this problem. Two strong candidates have emerged, the delinked Shafizadeh-Chin scheme, which is computationally attractive, and the Broido scheme, which is physically attractive. We have determined that the delinked scheme suffers a (mitigated) form of the one-step-scheme flaw. This is seen by comparing the normalized residual mass for the one-step and the delinked schemes. For the one-step scheme under fixed-temperature heating we have $z=e^{-\tau}$, and under variable-rate heating $z=e^{-v}$. It is easily shown that the delinked scheme gives exactly the same results. The Broido scheme, however, is completely different. In fact, it is a built-in feature of the Broido scheme that the char and volatile yields vary with the local temperature. Why is this important? Because it can be employed in models where a transition phenomenon is occurring. An excellent example is the smolder/spread transition phenomenon. Under some conditions, like low temperature, the charring pathway is more active than the volatile pathway. In this case smoldering is observed. Under different conditions the volatile pathway is preferred, in which case a gas-phase spreading flame will be observed. Hence, this competition is in fact necessary to decide the transition event.

Other examples where the char/volatile competition are important are ordinary flame spread 
models (wind-aided or wind-opposed) and the generation of volatiles in biomass gasification devices.

There is another feature of the Broido scheme that makes it attractive from the modeling standpoint. For a model to be useful it must be: (1) simple (understandable) and physically consistent with the available evidence, (2) complex and variegated enough to produce interesting, multi-faceted behaviors. In these respects the Broido model is quite satisfactory. We have used Activation-Energy-Asymptotics (AEA) in our analysis of these schemes because the methods suit, not because we have sought schemes for analysis by AEA. Interestingly, for the temperature range of ordinary pyrolysis, $500 \mathrm{~K} \leq T \leq 850 \mathrm{~K}$, the most important parameter of AEA, the nondimensional activation energy $\beta$, is in a nearly optimum range of values for maximum accuracy of the method. For larger and smaller $T, \beta$ decreases making the AEA procedure less accurate.

\section{CONCLUSIONS}

In this article we have examined three reaction schemes used to describe the pyrolysis and degradation of cellulosic materials. The Broido scheme is clearly the most versatile. This fact is sometimes misunderstood in the literature, particularly with respect to $\mu$, the maximum char yield. Some investigators have taken $\mu$ to represent a fixed char yield. Actually, $\mu$ is the maximum char obtainable for experiments performed at temperatures lower than $T_{c r}$ and giving $\alpha_{f} / \alpha_{c} \ll O(1)$. The actual char yield will always be smaller than $\mu$. Support for the Broido model is provided by Lewellen et al. [12], who performed experiments on cellulose at high heating rates. They reviewed the previous models, finding them unsatisfactory, then deduced a new one from their data. Ironically, their predictions can easily be reproduced by the Broido model for high heating rates although their model does not reduce to the Broido model for low heating rates. We conclude that the Lewellen-Peters-Howard model is not a new model but a limiting case of the more general Broido model.

One difficulty with the Broido model that has been mentioned in [4] occurs in variable oxygenconcentration environments. Though the Broido and delinked models can probably be recast to exhibit oxidation influences, it is not clear how this can be done for high or low oxygenconcentration environments. We recall that Ref. 4 added separate global cellulose and char oxidation reactions to the existing [1-3] global decomposition reaction.

Concerning the one-step reaction, we have shown here that it is useful when qualitative predictions are sought. Reasonable chemical parameters can be chosen based on the three-step models. It is a useful tool for understanding the variable-temperature heating rate curves, providing insight into the pyrolysis temperature question. In many respects, then, it describes some features of the more complex three-step mechanisms well. It is possible to generalize the one-step model by introducing a variable char yield instead of the fixed value. We can use the value suggested by Broido, $y_{c}(\infty)=\mu /\left\{1+\alpha_{T} / \alpha_{c}\right\}=\mu /\left\{1+\left(A_{T} / A_{c}\right) \exp \left[-\left(E_{T}-E_{c}\right) / R T\right]\right\}$ $=\mu /\left\{1+\kappa \exp \left[-\left(\epsilon_{T}-\epsilon_{c}\right) \beta(1-\theta) /[1-\sigma(1-\theta)]\right\}\right.$, where $\kappa=\left(A_{T} / A_{c}\right) \exp \left[-\left(E_{T}-E_{c}\right) / R T_{M A X}\right]$ and $\epsilon_{i}=E / E$, where $E$ is the activation energy for the overall decomposition reaction. Obviously, if $\left(\epsilon_{T}-\epsilon_{c}\right) \beta$ is small then $y_{c}(\infty)=\mu /(1+\kappa)$, and we shall return to the case of constant char yield.

We have quite convincingly demonstrated the absence of a single pyrolysis temperature, $T_{p}$. A 
unique value of $T_{p}$ exists only in the limit $E / R T_{M A X} \gg O(1)$. However, we have also demonstrated that the parameters of cellulose pyrolysis [6] produce nondimensional activation energies $\beta_{i}$ that are quite large. Hence, we expect that the condition of large $E / R T_{M A X}$ will be fulfilled, and that most woody and cellulosic materials will appear to possess a characteristic $T_{p}$, at least when the material is thermally thin. As already mentioned, for thermally thick materials the relevant issue is not whether we have certain fixed value of $T_{p}$, but what the status is of the instantaneous competition between the rates of formation of volatiles and char. This determines whether we have flame spread or smolder spread.

\section{NOMENCLATURE}

$A_{i} \quad$ Pre-exponential factor for $\mathrm{i}^{\text {th }}$ reaction

$D \quad D=t_{\text {HEAT }} f t_{K N N}$, a Damköhler number

$E_{i} \quad$ Activation energy for $\mathrm{i}^{\text {th }}$ reaction

$E_{1}(x)$ Exponential integral

$f_{i}(\theta) \quad f_{i}(\theta)=\exp \left[-\beta_{i}(1-\theta) /[1-\sigma(1-\theta)]\right.$, reaction-rate function

$g(t) \quad$ Heating rate function

$G$ Normalized heating rate, $G(\tau)=g(t) / g(0)$

$k_{i} \quad$ Kinetic rate constants

$R \quad$ Universal gas constant

$t_{K I N} \quad t_{K I N}=t_{C E L L}=\left[A_{C E L L} \exp \left(-E_{C E L V} / R T_{M A X}\right)\right]$

$t$ Time

$T$ Temperature

$\mathrm{X} \quad \mathrm{X}=t_{\text {eest } 1} / t_{\text {test } 2}$

$y_{i} \quad$ Species mass fraction, mass of specie $\mathrm{i}$ divided by initial sample mass (pure cellulose)

$\Upsilon \quad \Upsilon=T_{\text {rest } 1} / T_{\text {test } 2}$

$z \quad$ Normalized residual mass

\section{Greek}

$\alpha \quad \alpha=\alpha_{T}+\alpha_{c}$, sum of $\alpha^{\prime} s$ for char and tar steps

$\alpha_{i} \quad \alpha_{i}=k_{i}\left(T_{M A X}\right) / k_{C E L L}\left(T_{M A X}\right)=t_{K N N} d t_{i}\left(T_{M A X}\right)$

$\beta_{i} \quad \beta_{i}=\sigma\left(E_{i} / R T_{M A X}\right)$

$\gamma_{i} \quad \gamma_{i}=T / T_{M A X_{i}}$

$\epsilon_{i} \quad \epsilon_{i}=E / E_{C E L L}$

$\theta \quad \theta=\left(T-T_{o}\right) /\left(T_{M A X}-T_{o}\right)$, normalized temperature

$\Theta \quad \Theta=\beta(1-\theta)$, normalized stretched temperature

$\mu \quad$ Char yield

$\sigma \quad \sigma=1-T_{d} / T_{M A X}$

$v \quad$ Function defined in Eqs. (5)

$\tau \quad$ Nondimensional time, $\tau=t / t_{K T N}$

$\Omega \quad \Omega=\left|-H^{\prime}(1)\right| D^{-1}$

\section{Subscripts}

$\begin{array}{ll}A & \text { Active material } \\ c & \text { Char } \\ C E L L & \text { Cellulose } \\ c r & \text { Crossover value } \\ g & \text { Gas } \\ H E A T & \text { Pertaining to heating } \\ K I N & \text { Pertaining to kinetics } \\ M A X & \text { Maximum value } \\ o & \text { Ambient value } \\ p & \text { Pyrolysis value }\end{array}$


3D.L. Three-Step Delinked

\section{REFERENCES}

1. Wichman, I.S., and Atreya, A., "A Simplified Model for the Pyrolysis of Charring Materials," Combustion and Flame, 68, 231-247 (1987).

2. Villermaux, J., Antoine, B., Lede, J., and Soulignac, F., "A New Model for Thermal Volatilization of Solid Particles Undergoing Fast Pyrolysis," Chem. Eng. Sci., 41(1), 151-157 (1986).

3. Fredlund, B., "Modelling of Heat and Mass Transfer in Wood Structures During Fire," Fire and Safety Journal, 20, 39-70 (1993).

4. Kashiwagi, T., and Nambu, H., "Global Kinetic Constants for Thermal Oxidative Degradation of a Cellulosic Paper," Combustion and Flame, $\underline{88}$, 345-368 (1992).

5. Shafizadeh, F., and Chin, P.P.S., ACS Symposium Ser. 43,57 (1977).

6. Shafizadeh, F., "The Chemistry of Pyrolysis and Combustion," in The Chemistry of Solid Wood, R. Rowell (ed.), ACS, Washington, D.C., 1984.

7. Broido, A., and Nelson, M.A., "Char Yield on Pyrolysis of Cellulose, " Combustion and Flame, 24, 263-268 (1975).

8. DiBlasi, C., "Modeling and Simulation of Combustion Processes of Charring and NonCharring Solid Fuels," Prog. Energy Combust. Sci., 19, 71-104 (1993).

9. Lipska, A.E., and Parker, W.J., "Kinetics of the Pyrolysis of Cellulose in the Temperature Range $250-300^{\circ} \mathrm{C}$," J. Appl. Polym. Sci., 10, 1439-1453 (1966).

10. Wichman, I.S., and Melaaen, M., "Modeling the Pyrolysis of Cellulosic Materials," in Advances in Thermochemical Biomass Conversion, T. Bridgewater (ed.), Chapman and Hall, London, 1993.

11. Diblasi, C., "Analysis of Convection and Secondary Reaction Effects within Porous Solid Fuels Undergoing Pyrolysis," Comb. Sci. Tech., 90, 315-340 (1993).

12. Lewellen, P.C., Peters, W.A., and Howard, J.B., "Cellulose Pyrolysis Kinetics and Char Formation Mechanism," Sixteenth Symposium (International) on Combustion, The Combustion Institute, Pittsburgh, 1976, pp. 1471-1480. 
